\title{
Acidos grasos trans en aceites hidrogenados: implicaciones técnicas y nutricionales $\left(^{*}\right)$
}

\author{
Por D. Barrera-Arellano y J.M. Block \\ Lab. de Oleos e Gorduras. FEA/UNICAMP. Caixa Postal 6091. Campinas. SP-13081-970. Brasil. \\ * Trabajo presentado en el IV Congreso Latinoamericano sobre Procesamiento de Aceites. Nov/92. Rosario, Argentina.
}

\section{RESUMEN}

Acidos grasos trans en aceites hidrogenados: implicaciones técnicas y nutricionales.

Actualmente, las grasas y aceites son alimentos observados con mucha atención por los consumidores, por su estrecha relación entre ellos y la salud. Uno de los puntos todavía confusos y que ciertamente vendrá rápidamente a tornarse de dominio público, es la presencia de ácidos grasos trans en grasas hidrogenadas y productos que las contienen. En aceites y grasas de origen vegetal estos isómeros trans están ausentes, y son producidos durante la hidrogenación, este fenómeno es inevitable, pero puede ser controlado y mínimizado. La presencia de estos compuestos modifican tanto las características técnicas como también, se sospecha ocasionan problemas y alteraciones desde el punto de vista nutricional y de salud. En productos hidrogenados brasileños fueron encontrados valores elevados de ácidostrans (hasta 62\%), observándose un efecto notable en el contenido de sólidos (SFC) a bajas temperaturas y ninguna influencia en el punto de reblandecimiento «softening point». Posibles efectos nutricionales y de salud relacionados con ácidos grasos trans, también serán discutidos.

PALABRAS-CLAVE: Aceite hidrogenado - Acido graso - Evaluación nutricional - Información (artículo) - Isómero trans.

\section{SUMMARY}

Technical and nutritional implications of trans fatty acids in hydrogenated oils.

Customers are increasingly paying attention to fats and oils because they are strongly health related. Many points are still confusing about this, but certainly there is one that will soon become public concern: the presence of trans fatty acids in hydrogenated fats and their products. Natural fats and oils from vegetable origin lack this trans isomers. Eventhough the production of the trans isomers during hydrogenation is unavoidable it can be controlled and minimized. The presence of these components modify technical characteristics as well as introduce problems and alterations in health and nutritional aspects. High trans fatty acids values (up 62\%) were found in Brazilian products. A relevant effect in solid content (SFC) at low temperatures was observed but no influence in the softening point. Possible nutritional and health effects related to trans fatty acids will be discussed.

KEY-WORDS: Fatty acid - Hydrogenated oil - Information (paper) Nutritional evaluation - Trans isomer.

\section{INTRODUCCION}

Los aceites y grasas comestibles están formados predominantemente por triglicéridos (ésteres de 3 ácidos grasos monocarboxílicos y glicerol), de modo que los ácidos grasos presentes pueden encontrarse en diversas formas isoméricas (Gunstone y Norris, 1982).

Los ácidos grasos saturados poseen únicamente isómeros resultantes de ramificaciones en la cadena, este tipo de isómeros son poco comunes en aceites y grasas, mientras que en los ácidos grasos insaturados encontramos dos tipos de isomería: de posición y geométrica. En el primer caso se refiere a la posición del doble enlace en la cadena, siendo que estas uniones comúnmente se encuentran en los carbonos 9, 12 ó 15. En la isomería geométrica estos dobles enlaces, que impiden la rotación de los átomos de carbono, generan dos o más segmentos en la cadena, cuando están situados del mismo lado (isómeros cis) forman una molécula doblada. Cuando los segmentos se sitúan en posiciones opuestas, manteniendo la molécula recta (Figura 1), el isómero es llamado trans.

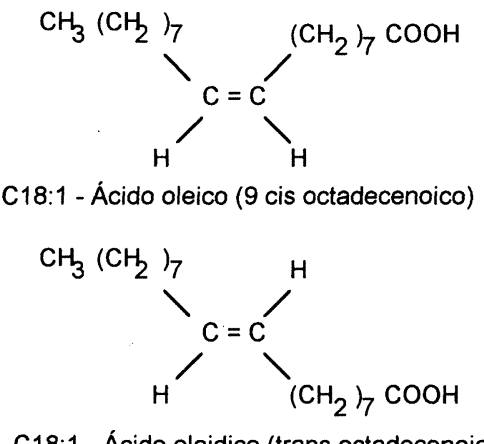

C18:1 - Ácido elaidico (trans octadecenoico)

Fig. 1

Isomería geométrica en ácidos grasos monoinsaturados (Gurr., 1986)

En ácidos grasos con más de un doble enlace pueden existir varias configuraciones. De esta manera, el ácido linoleico (C18:2) presenta los isómeros cis-cis, cis-trans y trans-trans, como se observa en la Figura 2 (Weiss, 1980).

En la naturaleza los ácidos grasos se encuentran con más frecuencia en la forma cis, siendo los isómeros trans prácticamente inexistentes en aceites y grasas de origen vegetal no refinados, mas estos isómeros pueden ser formados durante reacciones químicas, como la oxidación, que ocurre durante la extracción, refinación, almacenado o en la hidrogenación (Smith et al., 1978; Kochhar y Matsui, 


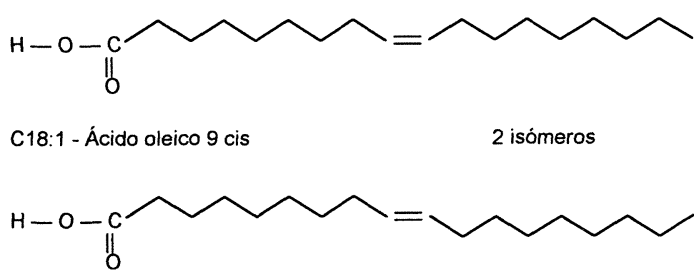

C18:1 - Ácido elaidico 9 trans

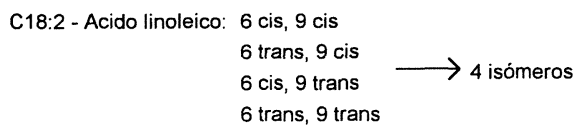

C18:3 - Ácido linolénico: $3,6,9$ cis $\longrightarrow 8$ isómeros

Fig. 2

Isómeros posibles en ácidos grasos C18 (Weiss, 1980).

1984). Estos compuestos están siendo motivo de discusión sobre sus efectos desde el punto de vista tecnológico y principalmente nutricional. Este trabajo resume los principales aspectos desde los dos puntos de vista, exponiendo los conceptos y tendencias más difundidas en esta área.

\section{HIDROGENACION}

Con algunas excepciones, los aceites vegetales en su forma natural no tienen las propiedades y características adecuadas para ser incorporadas en un gran número de alimentos. Por lo tanto, es necesaria una modificación que los torne plásticos en un amplio rango de temperaturas. Los métodos más usados para esta modificación, de las características fisicas de aceites, son hidrogenación, fraccionamiento, interesterficación y mezclado o «blending» (Nichols, 1989).

Entre los métodos utilizados en la modificación de la consistencia de aceites, la hidrogenación es el proceso industrial más ampliamente usado. Este proceso posee una versatilidad inherente, que hace posible la producción de una gran variedad de productos con características de plasticidad y comportamiento térmico específico (Patterson, 1983; Chrysam, 1985).

La composición y propiedades físico-químicas de las grasas hidrogenadas dependen del grado de hidrogenación de los aceites usados como materias primas, generalmente aceites con alto contenido de ácidos grasos insaturados. El aceite de soja, por ejemplo, que contiene aproximadamente $80 \%$ de ácidos mono y poliinsaturados, presenta un punto de fusión bajo y una estabilidad oxidativa inadecuados para algunas aplicaciones en alimentos, siendo así, el proceso de hidrogenación de esta materia prima posibilita el cambio de sus características, tornándolo adecuado para la industria productora de chocolates, helados, panificadoras, etc (Brisson, 1982).

La reacción de hidrogenación consiste en la adición de hidrógeno en los dobles enlaces de los ácidos grasos insa- turados, catalizada por un metal, originando su saturación. Esta reacción aparentemente simple, es en realidad una reacción muy compleja, que incluye, además de la saturación, otras reacciones paralelas. En el contacto con el catalizador (níquel es el más usado) algunos enlaces pueden adquirir la configuración trans o cambiar de posición, además de la formación de enlaces dobles conjugados. Todas estas reacciones originan cambios en la composición y características físicas del aceite, aumentando su punto de fusión y su estabilidad oxidativa (Patterson, 1983; Allen, 1981).

Como resultado de la hidrogenación, la composición de ácidos grasos del producto guarda poca o ninguna similaridad con la materia prima utilizada, esta diferencia es mayor cuanto mayor sea el grado de hidrogenación (Brisson, 1982). En general, en la formulación de margarinas, grasas comestibles (shortenings) y estearinas, intervienen en menor o mayor porcentaje los aceites hidrogenados (Templeman, 1977).

\subsection{Selectividad e isomerización}

La reacción de hidrogenación visa la reducción preferencial de ácidos grasos más insaturados con poca o ninguna formación de ácidos saturados. Esto implica una selectividad elevada de la reacción, que depende principalmente de las condiciones del proceso y tipo de catalizador (Allen, 1981; Coenen, 1981).

La Figura 3 muestra un gráfico de la hidrogenación de aceite de soja, donde la reducción de ácido linolénico (C18:3) produce ácido linoleico (C18:2) o isolinoleico (C18:2 trans), que se transforma en oleico (C18:1) o elaídico (C18:1 trans) y finalmente en esteárico (C18:0). Esta reacción, dependiendo de la selectividad, ocurre con uno o varios ácidos grasos al mismo tiempo, más con diferente velocidad de reacción (Allen, 1981).

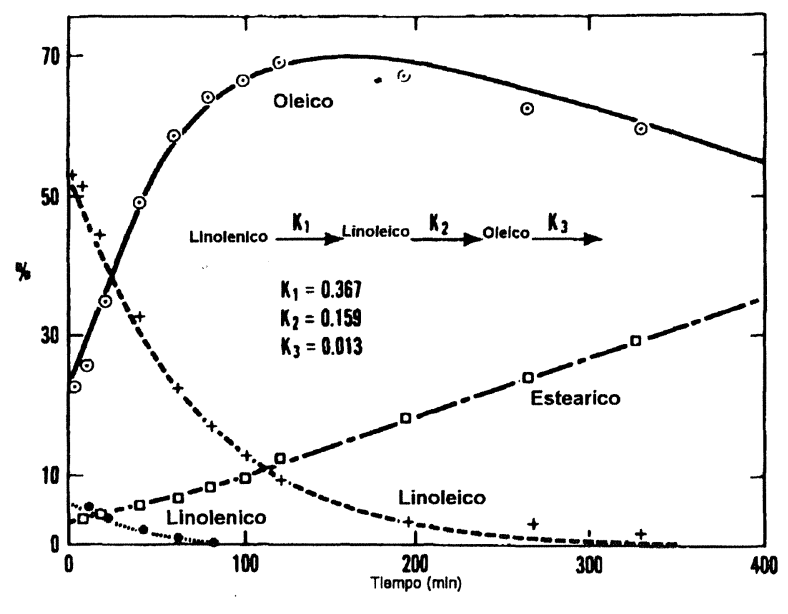

Fig. 3

Hidrogenación de aceite de soja. Efecto del tiempo de hidrogenación en la concentración individual de ácidos grasos. Condiciones de reacción: temperatura: $175^{\circ} \mathrm{C}$; presión de $\mathrm{H}_{2}$ : 15 psi; concentración del catalizador: 0,02\% Ni/peso del aceite; agitación: 600 rpm (Allen, 1978) 
El control rígido de las condiciones de la reacción como temperatura, presión, agitación, concentración y tipo de catalizador, permite hacer un diseño preciso del producto final, sin embargo las alteraciones en estas condiciones originarán cambios en la selectividad, velocidad de reacción e isomerización (Tabla I) (Young et al., 1986).

Tabla I

Efecto de las condiciones del proceso de hidrogenación sobre la selectividad e isomerización

\begin{tabular}{lcc}
\hline & \multicolumn{2}{c}{ Efecto Sobre } \\
\multicolumn{1}{c}{ Aumento de } & Selectividad & Isomerización \\
\hline Temperatura & $\uparrow$ & $\uparrow$ \\
Presión & $\downarrow$ & $\downarrow$ \\
Agitación & $\downarrow$ & $\downarrow$ \\
Concentración de Catalizador & $\uparrow$ & $\uparrow$ \\
\hline
\end{tabular}

(Young et al., 1986)

\section{ISOMEROS TRANS Y CARACTERISTICAS FUNCIONALES DE LAS GRASAS HIDROGENADAS}

Las margarinas y la mayor parte de las grasas vegetales de uso específico son sólidos plásticos homogéneos, formados por una masa de pequeños cristales, entre los cuales existe una porción considerable de aceite líquido (Greenwell, 1981).

Las características finales del producto dependen de la composición de ácidos grasos, del contenido de grasa sólida (CGS) y la distribuición sólido/líquido en determinados rangos de temperatura, del comportamiento o tendencias de cristalización, del punto de fusión de los triglicéridos y de las condiciones de proceso, tales como agitación y forma de enfriamiento (Greenwell, 1981; Wiedermann, 1978; Lefebvre, 1983).

Los ácidos grasos trans, formados durante la hidroge- nación, también están relacionados con las características finales de estas grasas. Este efecto se da porque su presencia influye en los procesos de fusión y cristalización, además de que son un factor determinante en el contenido de grasa sólida a ciertas temperaturas (Frankel, 1981; DeMan y DeMan, 1983; Busfield y Proschogo, 1990).

Los isómeros trans tienen puntos de fusión siempre mayores que los cis (Tabla II), por ejemplo, el ácido oleico tiene un punto de fusión de $16^{\circ} \mathrm{C}$ mucho menor que los $45^{\circ} \mathrm{C}$ para su isómero trans, el ácido elaidico. El ácido esteárico, que representa la forma saturada de $18 \mathrm{car}$ bonos funde a $73.1^{\circ} \mathrm{C}$ (UNICHEMA, 1987) y tiene un efecto determinante en el contenido de grasa sólida a altas temperaturas, mientras que el elaidico (C18:1 trans) lo tiene a temperaturas intermedias (Allen, 1981).

Además de aumentar el punto de fusión de las grasas, el contenido de isómeros trans altera la estabilidad térmica de las estructuras cristalinas y consecuentemente la textura de los productos (DeMan y DeMan, 1983; Busfield y Proschogo, 1990).

Los aceites y grasas son sustancias que presentan el fenómeno de polimorfismo, o sea, pueden cristalizar en varias formas. Esta propiedad se debe a las varias posibilidades de ordenación molecular de los triacilglicéridos en los cristales. Las 3 formas principales encontradas en grasas son $\alpha, \beta^{\prime}$ y $\beta$ que tienen puntos de fusión y estabilidad creciente (Gunstone y Norris, 1982).

Ha sido observado que muestras con elevada concentración de isómeros trans, muestran una mayor estabilidad de cristalización en la forma $\beta$ '. Esto indica que el contenido de trans puede ser un factor determinante en la relación de transición polimórfica de grasas hidrogenadas, debido a que los trans isómeros podrían generar un impedimento estérico para la formación de la estructura $\beta$.

Los resultados indican que los ácidos grasos trans influyen positivamente sobre la textura de las grasas hidrogenadas, una vez que las formas $\beta^{\prime}$ son preferidas en

Tabla II

Puntos de fusión de ácidos grasos y sus isómeros

\begin{tabular}{|c|c|c|c|}
\hline Símbolo & Nombre sistemático & Nombre común & Punto de fusión $\left({ }^{\circ} \mathrm{C}\right)$ \\
\hline C 12:0 & Dodecanóico & Laurico & 44,2 \\
\hline C 16:0 & Hexadecanóico & Palmitico & 63,1 \\
\hline C 18:0 & Octadecanóico & Estearico & 69,6 \\
\hline C 18:1(6 c) & 6c-octadecenóico & Petroselinico & 29 \\
\hline C 18:1(6t) & 6t-octadecenóico & Petroselaídico & 54 \\
\hline C 18:1 (9 c) & 9c-octadecenóico & Oleico & 16 \\
\hline C $18: 1(9 t)$ & 9t-octadecenóico & Elaidico & 45 \\
\hline C 18:1 (11c) & 11c-octadecenóico & cis-vacenico & 15 \\
\hline C 18:1 (11t) & 11t-octadecenóico & trans-vacenico & 44 \\
\hline C 18:2 (9c,12c) & 9c,12c-octadecadienóico & Linoleico & -5 \\
\hline C 18:2 (9c,12t) & 9c,12t-octadecadienóico & Linoelaidico & 28 \\
\hline C $18: 2(9 t, 11 t)$ & 9t,11t-octadecadienóico & & 54 \\
\hline C $18: 3(9 c, 12 c, 15 c)$ & 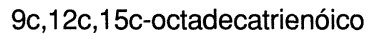 & Linolénico & -11 \\
\hline C $18: 3(9 t, 12 t, 15 t)$ & 9t,12t,15t-octadecatrienóico & & 30 \\
\hline C $18: 3(9 c, 11 t, 13 t)$ & 9c,11t,13t-octadecatrienóico & $\alpha$-eleostearico & 49 \\
\hline C $18: 3(9 t, 11 t, 13 t)$ & 9t, 11t,13t-octadecatrienóico & $\beta$-eleostearico & 71,5 \\
\hline
\end{tabular}

(UNICHEMA, 1987) 
estos productos, donde los cristales $\beta$ deben ser evitados, ya que dan una apariencia granulosa a los mismos (Naguib-Mostafa y DeMan, 1985).

Sobre los efectos de la cantidad de ácidos grasos trans, en relación al contenido de grasa sólida en grasas hidrogenadas de composición similar, fue observado que a mayor contenido de isómeros trans corresponden mayores cantidades de grasa sólida, así como más altos puntos de reblandecimiento (Tabla III y Figura 4) (Barrera-Arellano y Block, 1992).

Tabla III

Acidos grasos trans, contenido de grasa sólida (CGS) y punto de reblandecimiento (PR) en grasas hidrogenadas.

\begin{tabular}{|c|c|c|c|c|c|c|c|c|}
\hline \multirow[b]{2}{*}{ Muestra* } & \multirow[b]{2}{*}{ Trans \% } & \multirow[b]{2}{*}{$\operatorname{PR}\left({ }^{\circ} \mathrm{C}\right)$} & \multicolumn{6}{|c|}{ CGS (\%) } \\
\hline & & & 10 & 20 & 25 & 30 & 35 & 37.5 \\
\hline 1 & 25.5 & 55.8 & 36.2 & 24.1 & 23.5 & 21.6 & 17.1 & 14.7 \\
\hline 2 & 30.1 & 54.0 & 41.4 & 24.8 & 23.4 & 21.6 & 16.6 & 14.0 \\
\hline 3 & 35.6 & 53.9 & 46.1 & 29.3 & 27.0 & 24.4 & 16.9 & 13.3 \\
\hline 4 & 38.8 & 52.5 & 53.6 & 34.5 & 31.6 & 27.9 & 18.3 & 13.5 \\
\hline 5 & 44.8 & 56.0 & 54.8 & 34.4 & 32.0 & 29.0 & 20.7 & 17.2 \\
\hline 6 & 50.6 & 55.8 & 61.2 & 38.5 & 34.8 & 31.1 & 21.4 & 17.4 \\
\hline 7 & 51.0 & 55.8 & 66.1 & 41.6 & 37.6 & 33.8 & 22.7 & 17.5 \\
\hline
\end{tabular}

( ${ }^{*}$ ) Composición en ácidos grasos similar, índice de iodo $=70$. (Barrera-Arellano y Block, 1992)

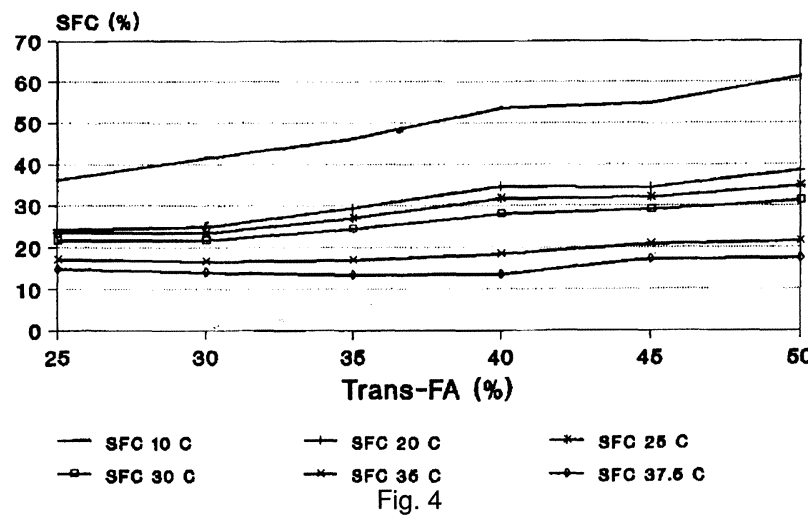

Efecto de los ácidos grasos trans sobre el contenido de grasa sólida en grasas hidrogenadas (Barrera-Arellano \& Block, 1992)

Desde el punto de vista tecnológico, la formación de ácidos grasos trans, a través de la hidrogenación parcial es deseable, ya que proporciona productos con puntos de reblandecimiento y rango plástico similar a los observados en grasas animales, y con características deseables para su incorporación en alimentos (Brisson, 1982).

\section{PRESENCIA DE ACIDOS GRASOS TRANS EN ALIMENTOS}

En grasas de origen animal y productos lácteos, los isómeros trans son compuestos naturales formados por biohidrogenación, con las enzimas producidas por la flora microbiana presente en el rúmen de los animales poligástricos, incluyendo entre éstas isomerasas e hidrogenasas (Kochhar y Matsui, 1984; DeMan y DeMan, 1983; Sommerfield, 1983).

Los aceites vegetales parcialmente hidrogenados, margarinas y grasas comestibles (shortenings) son ampliamente utilizados como materias primas de numerosos productos alimentícios, por lo tanto, los ácidos grasos trans pueden estar presentes en una gran variedad de alimentos.

El contenido de isómeros trans de varios tipos de alimentos es presentado en la Tabla IV. En mantequilla han sido reportados rangos entre 4.6 y $7.8 \%$ (Parodi, 1976), valores medios de $4 \%$ (Smith et al., 1978) y variaciones entre $4.9 \%$ en verano y $6.5 \%$ en invierno (DeMan y DeMan, 1983).

Tabla IV

\section{Contenido de ácidos grasos trans en alimentos}

\begin{tabular}{lcc}
\hline Producto & $\begin{array}{c}\text { Contenido de Grasa Total } \\
(\%)\end{array}$ & $\begin{array}{c}\text { Acidos grasos trans } \\
(\%)\end{array}$ \\
\hline Panes & 2 & $10-28$ \\
Pasteles & $11-26$ & $10-24$ \\
Pudines & $3-18$ & $31-36$ \\
Papas Fritas & $7-15$ & $5-35$ \\
Botanas (Snacks) & 36 & $14-34$ \\
Mantequilla & 82 & $1-7$ \\
Margarinas duras & 81 & $18-36$ \\
Margarinas cremosas & 81 & $0-21$ \\
Grasas hidrogenadas & 99 & $13-37$ \\
\hline
\end{tabular}

(Enig et al., 1983)

En grasas hidrogenadas han sido encontrados valores entre 9.4 y $28.6 \%$ (Rabascall y Riera, 1988). En Brasil, valores medios de $20,7 \%$ en margarinas cremosas, $32.2 \%$ en margarinas duras, $23.1 \%$ en cremas vegetales y $39.7 \%$ en grasas hidrogenadas (Soares y Franco, 1990), y rangos entre 12.3 - $38.1 \%$ en margarinas, $15.9-25.5 \%$ en cremas vegetales, $0-62.1 \%$ en grasas hidrogenadas y $4-6.2 \%$ en mantequilla (Tabla V) han sido reportados (Block, 1992).

Según Enig et al. (1983) el consumo medio de ácidos trans en Estados Unidos es aproximadamente 12g/dia, siendo $4.8 \%$ de origen animal y $95.2 \%$ originarios de aceites vegetales parcialmente hidrogenados, consumidos indirectamente en alimentos preparados. Esta cantidad de ácidos trans representan $8 \%$ de la grasa ingerida (Brisson, 1982; Sundram, 1992). En Brasil, no existen datos sobre el consumo de grasas hidrogenadas, mas se estima que el consumo de margarinas alcanza $2.27 \mathrm{Kg} /$ persona-año (Anónimous, 1992b).

\section{EFECTOS NUTRICIONALES}

Los ácidos grasos tienen varias funciones fisiológicas, actúan como fuentes de energía, como componentes de biomembranas y como precursores de las prostaglandinas (Horrobin, 1992).

El aumento de la ingestión de ácidos grasos trans, a 
través de grasas hidrogenadas, ha despertado un gran interés de los investigadores, principalmente con respecto a sus aspectos metabólicos, incluyendo digestibilidad, absorción, acumulación en tejidos, catabolismo e incorporación en membranas. Debido a la importancia de las funciones de los ácidos grasos en el organismo humano, un gran número de investigaciones han sido realizadas en los últimos años, con el objetivo de evaluar los efectos de los ácidos grasos trans sobre estas funciones (Houstmuller, 1978; Anonimous, 1992). En este sentido, el ácido elaidico (C $18: 1$ trans), que representa aproximadamente 15\% de los trans isómeros encontrados en grasas hidrogenadas (Brisson, 1982), ha sido intensivamente estudiado.

Tabla V

Contenido de ácidos grasos trans en margarinas y grasas hidrogenadas*

\begin{tabular}{lllc}
\hline Pais & $N^{\circ}$ Muestras & Tipo de producto & $\begin{array}{c}\% \text { Trans } \\
\text { isómero }\end{array}$ \\
\hline USA & 26 & Marg. suave & $10.7-21.0$ \\
& 60 & Marg. dura & $14.8-30.1$ \\
& 13 & Marg. suave & $6.8-17.6$ \\
& 24 & Marg. dura & $15.9-31.0$ \\
Japón & 07 & Grasa hidrog. & $8.7-35.4$ \\
& 15 & Marg. suave & $13.7 \mathrm{a})$ \\
Alemania & 26 & Grasa hidrog. & $14.7 \mathrm{a})$ \\
& 60 & Marg. suave/dura & $0-12.9$ \\
Italia & 24 & Grasa hidrog. & $0-19.9$ \\
& 07 & Marg. suave & $0.6-21.2$ \\
Inglaterra & 07 & Marg. dura & $0.4-34.6$ \\
Dinamarca & 18 & Marg. suave/dura & $4.1-42.3$ \\
Brasil & 30 & Marg. suave/dura & $2.6-29.0$ \\
& 21 & Marg. suave & $14.4-31.3$ \\
& 12 & Marg. dura & $25.0-42.9$ \\
& 09 & Creme vegetal & $14.1-31.3$ \\
& 03 & Grasa hidrog. & $37.8-42.3$ \\
& 09 & Creme vegetal & $15.9-25.1^{* *}$ \\
& 14 & Marg. suave/dura & $13.4-38.1^{* *}$ \\
& 11 & Grasa hidrog. & $26.3-47.1^{* *}$ \\
\hline
\end{tabular}

* (Soares y Franco, 1990)

* * (Block, 1992)

a) valores medios

Los efectos de la presencia de ácidos grasos trans en aceites y grasas fueron investigados por un Comité Especial de Salud del Canadá (1980), concluyendo que:

1. Los ácidos grasos trans poseen propiedades similares a las de los ácidos grasos saturados.

2. Los ácidos grasos trans aumentan los síntomas de deficiencia de ácidos grasos esenciales.

3. Las propiedades arterogénicas de los ácidos trans son semejantes a las de los ácidos grasos saturados.

4. Los ácidos grasos trans pueden aumentar el peso de los riñones y el nivel de lípidos en el hígado y reducen el contenido del ácido araquidónico.

5. Los ácidos grasos trans son fácilmente absorbidos y metabolizados (DeMan et al., 1992).

\subsection{Absorción e incorporación en tejidos y mem-} branas.

Los ácidos grasos trans, en su mayoria, son absorbidos, transportados e incorporados en los téjidos de la misma manera que los isómeros cis (Brisson, 1982; Wood, 1979; Kinsella et al., 1981; Zevenbergen et al., 1988). Sin embargo, existen algunas diferencias en el grado de incorporación en triacilglicéridos simples o complejos, además de la velocidad con que ellos son metabolizados. Su incorporación en los tejidos depende de su concentración dietética, tiempo de la dieta, tipo de tejido e isómero (Wood, 1979; Schrijver y Privet, 1982; Astorg y Chevalier, 1987).

Los ácidos grasos trans, debido a su estructura, tienen más parecido con los ácidos grasos saturados que con los isómeros cis, teniendo puntos de fusión más elevados y son esterificados preferencialmente en las posiciones 1 y 3 (Beare-Rogers et al., 1979).

A pesar de que los isómeros cis son incorporados preferencialmente en fosfolípidos estructurales y funcionales, los ácidos trans también son incorporados en triacilglicéridos y fosfolípidos de las membranas biológicas, cambiando con esto su composición. Más, el efecto de estos ácidos grasos sobre la funcionalidad de las membranas es actualmente una pregunta sin respuesta (Zevenbergen et al., 1988; Reiser, 1981).

Con respecto a la absorción, ha sido reportado que el coeficiente de absorción de ácidos grasos trans es de 95\%, y depende de su concentración en la dieta (Sommerfield, 1983; Kinsella et al., 1981).

\subsection{Fuente de energía celular}

Los ácidos grasos trans ingeridos y absorbidos son transportados a las células donde pueden ser utilizados como fuentes de energía o depositados en los tejidos para utilización futura. Los ácidos grasos usados como fuente de energía son degradados por una serie de reacciones catalizadas por enzimas, siendo fragmentados progresivamente en grupos de Acetil CoA, a través de la $\beta$-oxidación, siendo finalmente oxidados vía ciclo de Krebs (Brisson, 1982; Reiser, 1981).

Lawson y Kummerov (1979) estudiaron algunos isómeros del ácido oleico con relación a su metabolismo en mitocondrias, concluyendo que los ácidos grasos cis son oxidados y metabolizados más rápidamente que los trans, siendo que la velocidad aumenta dependiendo de la localización del doble enlace. Así, el ácido vacénico-trans (isómero posicional y geométrico del ácido oleico), que representa la mayor proporción de isómeros trans en grasa de leche y grasas animales, es catabolizado a nivel celular de manera similar al oleico y no al elaídico (trans), presentando también menor tendencia de acumulación en el músculo cardiaco que el isómero trans.

\subsection{Acidos grasos esenciales}

Los ácidos grasos esenciales son componentes funda- 
mentales de todas las membranas celulares, regulando sus funciones y metabolismo. La flexibilidad y fluidez de las membranas dependen del nivel de ácidos grasos esenciales disponibles en las mismas, con participación en la selección de sustancias que entran y salen de las células, así como en las respuestas a impulsos nerviosos, funcionales y hormonales. Otra función importante de los ácidos grasos esenciales es que son los precursores de prostaglandinas E-1 y E-3, además de otros eicosanoides, que regulan el comportamiento de las células y tejidos. Así siendo, la deficiencia de ácidos grasos esenciales puede causar serios problemas metabólicos (Horrobin, 1992; San Juan, 1992).

Para el funcionamiento normal de las células y tejidos animales son considerados esenciales los ácidos linoleico (C18:2 $\omega$-3) y $\gamma$-linolénico (C18:3 $\omega$-3).

El ácido linolénico no tiene actividad metabólica, pero es necesario, ya que es transformado en compuestos biológicamente activos. En nuestro organismo existe una etapa de conversión del ácido linoleico en $\gamma$-linolénico catalizada por la enzima desaturasa, cuya actividad puede ser perjudicada por la presencia de isómeros trans (Kinsella et al., 1981; San Juan, 1992).

En la Figura 5 pueden ser observados los sitios de posibles interferencias de los ácidos grasos trans de la dieta en algunas etapas del metabolismo del ácido linoleico. Este efecto se da sobre las enzimas desaturasas que actúan en la conversión del ácido linoleico y araquidónico en prostaglandinas (Kinsella et al., 1981). Los isómeros trans debido a sus características estructurales son capaces de afectar las reacciones de desaturación y alargamiento, este efecto inhibitorio supone la competición de los ácidos trans con substratos comunes en las reacciones del metabolismo de ácidos esenciales, y siendo los productos finales de estructura diferente, entonces pueden ocurrir cambios en la composición de las membranas y prostaglandinas (Schrijver y Privet, 1982; Holman y Mahfouz, 1981; Emken, 1981; Holman, 1985).

Factores como la ingestión insuficiente de ácido linoleico, acompañada por la presencia de grandes cantidades

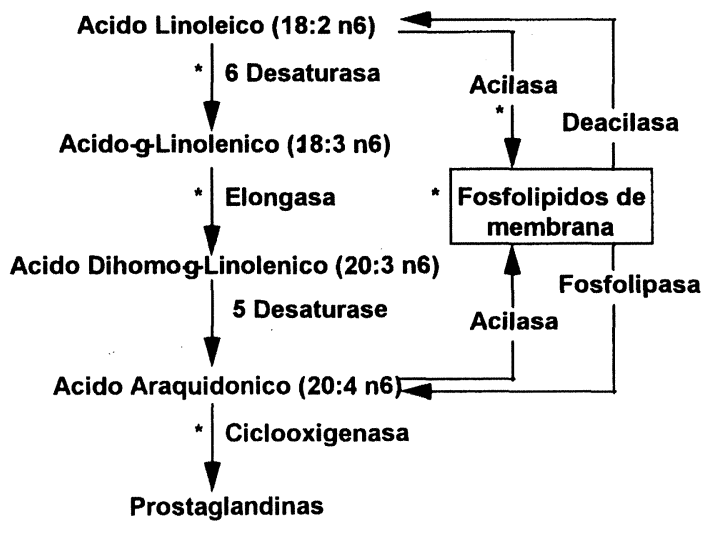

Fig. 5

Vías metabólicas del ácido linoleico hasta prostaglandinas (Kinsella et al., 1981). de isómeros trans, pueden ocasionar cambios metabólicos, haciendo que los ácidos trans actúen como substratos alternativos, generando ácidos grasos no esenciales, que formarían prostaglandinas de efecto desconocido o impredecible. De acuerdo con estos estudios, se puede concluir que los ácidos grasos trans afectan la disponibilidad de ácidos grasos esenciales de dos maneras:

- Sustituyendo los ácidos grasos esenciales en los tejidos, $y$

- Bloqueando el metabolismo, actuando como inhibidores (Gurr, 1986).

De estas observaciones, ha sido sugerida la necesidad de mayor ingestión de ácido linoleico en dietas que contengan grasas hidrogenadas (Astorg y Chevalier, 1987), ya que ha sido observado que los efectos negativos de los ácidos grasos trans sobre el metabolismo de ácidos esenciales no ocurre, cuando el ácido linoleico es ingerido en cantidades suficientes junto con los trans isómeros (Houstmuller, 1978; Kinsella et al., 1981; Zevenbergen et al., 1988; Beare-Rogers et al., 1979; Reiser, 1981). Dietas incluyendo $2 \%$ de ácido linoleico y $20 \%$ de trans isómeros no presentaron efectos adversos en la síntesis de prostaglandinas, funcionalidad de membranas, agregación de plaquetas y oxidación de ácidos grasos. Indicando que siendo $2 \%$ un nivel común de ingestión de ácido linoleico y el consumo de ácidos grasos trans de $8 \mathrm{~g} / \mathrm{dia}$, no hay razón para dudas en relación a la seguridad del proceso de hidrogenación (Zevenbergen et al., 1988).

\subsection{Arteriosclerosis}

La arterioesclerosis se caracteriza por los depósitos de lipidos en las paredes arteriales, siendo la mayor causa de enfermedades coronarias. La formación de estos depósitos está asociada al curso normal de la vida, siendo su causa desconocida. Algunos factores dietéticos como el exceso de vitamina $D$, colesterol y ácidos grasos trans, pueden estar relacionados con la aceleración del desenvolvimiento de esta alteración metabólica en humanos (Brisson, 1982; Kummerow, 1981).

El papel de los ácidos grasos trans en relación a la arterioesclerosis no está bien definido, existiendo mucha polémica en este sentido y sobre todo con las relaciones con el colesterol. Algunos experimentos indican que los isómeros trans pueden aumentar los niveles de colesterol sanguíneo (Anonimous, 1990), entre tanto otros autores alegan que estos isómeros no están relacionados con el colesterol sanguíneo, principalmente en dietas con contenido elevado de poliinsaturados (Brisson, 1982; Mattson et al., 1975).

La American Societies for Experimental Biology en 1985, publicó un artículo de consenso en el cual eliminan los ácidos grasos trans como factor que eleva el colesterol sanguíneo (DeMan et al., 1992).

En publicación reciente son comentados los estudios de Mensink y Katan, que se refieren a los efectos de los ácidos grasos trans en dietas de hombres y mujeres sanos, concluyendo que estos compuestos son hipercolesterolémicos, y que aumentan los niveles de lipoproteinas de baja 
densidad (LDL). Mas Emken, investigador del USDA, critica la metodologia de este estudio, porque los ácidos grasos isomerizados son totalmente diferentes a los presentes en grasas hidrogenadas, además de que según Hunter el tiempo del estudio es considerado muy corto (Anonimous, 1990). A pesar de estas observaciones, existe un consenso en relación a la ingestión de isómeros trans, sugeriendo prudencia con respecto a estos compuestos, sobre todo en personas con riesgo de arterioesclerosis.

\section{CONCLUSIONES}

La seguridad de la ingestión de ácidos grasos trans ha sido cada vez más discutida, y a pesar de las controversias surgidas, sería recomendable una disminución. Varias alternativas han sido propuestas, como la formulación de grasas hidrogenadas sin trans isómeros por «blending», de grasas completamente saturadas y aceites vegetales, o grasas plásticas producidas por interesterificación y fraccionamiento.

Modificaciones futuras de la legislación, posiblemente incluirán mayores informaciones para los consumidores, tanto sobre las cantidades presentes de estos ácidos grasos, como su inclusión como saturado para efecto del cálculo de la relación saturados/insaturados.

Seguramente, en los próximos meses y años presenciaremos un aumento en el número de investigaciones, publicaciones y discusiones sobre estos compuestos, tanto en los campos tecnológico y nutricional, que nos indiquen de forma más concreta los efectos de la presencia de los ácidos grasos trans en alimentos, ya que hasta la fecha, a pesar del gran número de informaciones disponibles, todavía no se ha llegado a tener un panorama claro sobre el asunto.

\section{BIBLIOGRAFIA}

Allen, R.R. (1978).- «Principles and catalysts for hydrogenation of fats and oils».-J. Am. Oil Chemists'Soc. 55, 792-795.

Allen, R.R. (1981).-«Hydrogenation»-- J. Am. Oil Chemists'Soc. 58, 166169.

Anonimous (1990).-«Netherlands study puts trans in spot light again».Inform 1, 875-878.

Anonimous (1992a).-«Session focuses on effects of trans».-Inform 3, 788.

Anonimous (1992b).-«Margarines or spreads?».-Oleos e Grãos 8, 32.

Astorg, P.O. y Chevalier, J. (1987).-« Polyunsaturated fatty acids in tissues of rats fed trielaidin and high or low levels of linolenic acid».-Lipids 22, 1025-1030.

Barrera-Arellano, D. y Block, J.M. (1992).-«Effect of trans-fatty acids on the melting behavior of hydrogenated fats".-Inform 3, 523,

Beare-Rogers, J.L., Gray, B. y Hollywood, R. (1979).-«The linoleic acid and trans fatty acids of margarines".-J. Am. Clin. Nutr. 32, 1805-1809.

Block, J.M. (1992).-«Comportamento Térmico de Gorduras Técnicas Produzidas no Brasil».-Tese (mestr.), Dept. de Tecnología de Alimentos. Universidade Federal de Santa Catarina, Florianópolis, S.C. Brasil.

Brisson, G.J. (1982).-«Lipids in Human Nutrition».-2nd Ed.-MTP Press, Lancaster.

Busfield, D.K. y Proschogo, P.N. (1990).-«Hydrogenation of palm stearine: changes in chemical composition and thermal properties".- J. Am. Oil Chemists'Soc. 67, 176-181.
Chrysam, M.M. (1985).-«Table Spreads and Shortenings» en «Bailey's Industrial Oil and Fat Products» 4nd Ed. p.41.-Daniel Swern (Ed.).Wiley Intercience Publ., New York.

Coenen, J.W.E. (1981).-«Selectivity in fat hydrogenation».- Riv. Ital. Sostanze Grasse 58, 445-452.

DeMan, L. y DeMan, J.M. (1983).-«Trans fatty acids in milkfat».-J. Am. Oil Chemists'Soc. 60, 1095- 1098.

DeMan, J.M., DeMan, L. y Yada, R. (1992).-«Nutritional aspects of fats».Dept. of Food Science, University of Guelph, Ontario, Canadá. Personal paper.

Emken, E.A. (1981).-«Influence of trans-9-, trans-12-, and cis-12-octadecenoic acid isomers on fatty acid composition of human plasma lipids".Progress in Lipid Reserch 20, 135-141.

Enig, M.G., Pallansch, L.A., Sampugna, J. y Keeney, M. (1983).-«Fatty acid composition of the fat in selected food itens with emphasis on trans components".-J. Am. Oil Chemists'Soc. 60, 1788-1795.

Frankel, G. (1981).-«Methods for analysis of processed soya oil».- J. Am. Oil Chemists'Soc. 83, 210-215.

Greenwell, B.A. (1981). -«Chilling and crystallization of shortenings and margarines".-J. Am. Oil Chemists'Soc. 58, 206-207.

Gunstone, F.D. y Norris F.A. (1982).-«Lipids in Foods: Chemistry, Biochemistry and Technology".-Pergamon Press, Oxford.

Gurr, M.I. (1986).-«Role of Fats in Food and Nutrition».-Elsevier Applied, London.

Holman, R.T. (1985).-«Influence of Hydrogenated Fats on the Metabolism of Polyunsaturated Fatty Acids» en «The Role of Fats in Human Nutrition» p.48. -F.B. Padley and J. Podmore (Ed.).-Ellis Harwood, England.

Holman, R.T. y Mahfouz, M.M. (1981).-«Cis and trans-octadecenoic acids as precursors of polyunsaturated acids".-Progress in Lipid Research 20 151-156.

Horrobin, D.F. (1992).-«Nutricional and medical importance of gamma-linolenic acid".-Progress in Lipid Research 31, 163-194.

Houstmuller, U.M.T. (1978).-«Biochemical aspects of fatty acids with trans double bonds".-Fette Seif. Anstrichm. 80, 162-169.

Kinsella, J.E., Bruckner, G., Mai, J. y Shimp, J. (1981).-«Metabolism of trans fatty acids with emphasis on the effects of trans, trans-octadecadienoate on lipid composition, essential fatty acid, an prostaglandins: an overview».-Am. J. Clin. Nutr. 34, 2307-2318.

Kochhar, S.P. y Matsui, T. (1984).-«Essencial fatty acids and trans contents of some oils, margarine and other food fats".- Food Chem. 13, 85-101.

Kummerow, F.A. (1981).-«The possible involvement of dietary fats in atherosclerosis".-Progress in Lipid Research 20, 743-746.

Lawson, L. G. y Kummerow, F. (1979).- « $\beta$ oxidation of the coenzyme A esters of vaccenic, elaidic and petroselaidic acids of rat heart mitochondria".-Lipids 14, 502.

Lefebvre, J. (1983).-«Finished product formulation».-J. Am. Oil Chemists'Soc. 60, 295-300.

Mattson, F.H., Hollenbach, E.J. y Kligman, A.M. (1975).-«Effect of hydrogenated fat on the plasma cholesterol and triglyceride levels of man».-Am. J. Clin. Nutr. 28, 726-731.

Naguib-Mostafa, A. y DeMan, J.M. (1985).-«P.olymorphism of hydrogenated canola oil».-J. Am. Oil Chemists'Soc. 62, 756-759.

Nichols, B.W. (1989).-«Processed oil and fat products».- Food Chem. 33, 27-31.

Parodi, P.W. (1976).-«Composition and structure of some consumeravaiable edible fats".-J. Am. Oil Chemists'Soc. 53, 530-534.

Patterson, H.B.W. (1983).-«Hydrogenation of fat and oils».-Applied Science Publishers, London.

Rabascall, N.H. y Riera, J.B. (1988).-«Contenidos de isómeros de los ácidos grasos en margarinas".-Grasas y Aceites 39, 348-352.

Reiser, R. (1981).- «Nutrition and metabolism of trans fatty acids».- Riv. Ital. Sostanze Grasse 53, 565-570.

San Juan, P.M.F. (1982).-«Estudio de la presencia del ácido gamma-linolénico en los aceites vegetales».- Alimentaria 3, 50-52. 
Schrijver, R. y Privet, O.R. (1982).-«Interrelationship between dietary trans iatty acids and 6- and 9- desaturases in the rat».- Lipids 17, 27-34.

Smith, L.M., Dunkley, W.L., Franke, A. y Daikiri, T. (1978).- «Measurement of trans and other isomeric unsaturated fatty acid in butter and margarine».-J. Am. Oil Chemists'Soc. 55, 257-261.

Soares, L. M. V. y Franco, M. R. B. (1990).-«Níveis de trans-isomeros e composição de ácidos graxos de margarinas nacionais e produtos hidrogenados semelhantes».-Cienc.Tecnol. Aliment. 10, 57-71.

Sommerfield, M. (1983).-« Trans unsaturated fatty acids in natural products and processed foods".- Progress in Lipid Research 22, 221-233.

Sundram, K. (1992).-«Trans fatty acids - a summary».-Palm Oil Developments 16, 17.

Templeman, G.J. (1977).-«Evaluation of several pulsed NMR techniques for solids-infat determination of commercial fats».-J. Food Sc. 42, 432-435.

UNICHEMA (1987).- «Fatty acid data book».-2nd Ed.-Germany.

Weiss, T.J. (1980).-«Food oils and their uses».-2nd Ed.-Avi Publishing Company, Chichester.

Wiedermann, L.H. (1978).-«Margarine and margarine oil, formulation and control».-J. Am. Oil Chemists'Soc. 55, 823-829.

Wood, R. (1979).-«Incorporation of dietary cis and trans octadecenoate isomers in the lipid classes of various rat tissues".- Lipids 14, 975-982.

Young, F.V.K., Poot, C., Biernoth, E., Krog, N., O'Neill, L.A. y Davidson, J. (1986).-«Processing of fats and oils» en «The lipid handbook» p. 178, F.D. Gunstone, J.L. Harwood and F.B. Padley (Ed.).-Chapman and Hall, London.

Zevenbergen, J.L., Houtsmuller, U.M.T. y Gottenbos, J.J. (1988).- «Linoleic acid requeriment of rats fed trans fatty acids».- Lipids $23,178-186$.

(Recibido: Enero 1993) 